\title{
Evoluzione della ricerca scientifica nell'ambito della biologia umana: abbiamo raggiunto il momento in cui dobbiamo fermarci e riflettere?
}

Giornale di Tecniche Nefrologiche e Dialitiche 2019, Vol. 3I(I) 37-39

(C) The Author(s) 2019

Article reuse guidelines:

sagepub.com/journals-permissions DOI: 10.1 | 177/0394936219836656 journals.sagepub.com/home/gtn

\section{(S)AGE}

\author{
Antonio Barracca', Patrizia Francesca Patricelli2, \\ Maria Rinaldi Milani ${ }^{3}$ e Giuseppe Quintaliani ${ }^{4}$
}

\begin{abstract}
Evolution of scientific research in human biology. Have we reached a point where we have to stop and reflect?

No one wants to question the scientific method, which is the method science uses to proceed and expand our knowledge of the reality. However, we can ask ourselves if scientific research has limits that we cannot overcome? In 1942, Julius Robert Oppenheimer, who ran the Manhattan project to develop the first atomic bomb, was the first to understand that the limits should not be overcome in scientific research. With the discovery of the molecular structure of the nucleic acid, and its meaning in the transmission of information in living beings, we have entered the knowledge of the "mysteries" of life. We have learned to read DNA, to write it and to modify it, cutting defective parts with the CRISPR/Cas9 technique. With genetic editing we can not only avoid many diseases, but also modify the DNA sequence in future generations. Have we reached a point where we must stop? Perhaps we must begin to consider that science must progress together with philosophical and ethical thought in the interest of the community.
\end{abstract}

\section{Keywords}

Scientific research, DNA, Human genome, Genetic editing, CRISP /Cas9

Correva l'anno 1300 e nel canto XXVI dell'Inferno della Divina Commedia, Dante fa parlare Ulisse che si rivolge così ai suoi compagni: "Considerate la vostra semenza: fatti non foste a viver come bruti, ma per seguir virtute e canoscenza". La storia dell'umanità, dell'uomo, è incentrata su questo anelito, la conoscenza. A cominciare dalla narrazione biblica che ci fa capire come per Adamo ed Eva il bisogno della conoscenza fu grande tanto da portarli a cogliere la mela dall'albero del bene e del male. La religione, la filosofia e poi la scienza hanno occupato il mondo della conoscenza. Chi siamo, che posto abbiamo nell'universo, che senso diamo alla nostra vita, sono tutte domande che l'uomo da sempre si pone e che continua a porsi accomunando in questo scienziati e filosofi. Prima dell'illuminismo la terra era al centro dell'universo e l'uomo era al centro della terra, come simbolo del progetto divino. Poi arrivò Copernico ${ }^{1}$ che immaginò il sole al centro del sistema solare, come già aveva sostenuto Aristarco di Samo, ${ }^{2}$ e la terra un semplice pianeta. Tutto cominciò a cambiare perché serviva un metodo per interpretare il mondo e questo ci è stato dato da Galileo, che ci ha raccontato che la filosofia scritta nel grande libro dell'universo non si poteva capire se non si fosse conosciuta la lingua ed i caratteri con i quali è scritta.

\footnotetext{
'Medico, specialista in Nefrologia e Urologia, Cagliari, Italy Medico, specialista in Nefrologia, Centro Dialisi, Ospedale SS. Trinità, ASSL, Cagliari, Italy

${ }^{3}$ Esperta in Web e Digital Marketing in Sanità

${ }^{4}$ Segretario Fondazione italiana del Rene. Editor www.renalgate.it

Corrispondenza:

Antonio Barracca, Medico, specialista in Nefrologia e Urologia, Viale Merello, 74, 09123, Cagliari, Italy.

Email: barraccaantonio@gmail.com
} 
Questo libro è scritto in lingua matematica. Ovvero serviva, e ci è stato dato, il metodo scientifico, cioè la modalità attraverso la quale la scienza procede per raggiungere una conoscenza della realtà. Ma la scienza, la ricerca scientifica, ha spostato l'obbiettivo della sua azione. Per il pensiero scientifico, la conoscenza non specula più per sapere qual è il nostro posto nell'universo, ma l'obbiettivo è invece conoscere com'è fatto l'universo. Com'è composta la materia fin nei suoi componenti più piccoli. La fisica ha aperto la strada a questa conoscenza, la fisica atomica. A Los Alamos, nel 1942, Julius Robert Oppenheimer $^{3}$ dirigeva il progetto Manhattan per sviluppare la prima bomba atomica. Fu il primo a capire quali potevano essere i limiti da non superare nella ricerca scientifica. Allo scoppio della bomba di Hiroshima fu consapevole della propria parte di responsabilità. "I fisici hanno conosciuto il peccato", fu il suo commento, che lo portò ad uscire dal progetto e a non partecipare allo sviluppo della bomba all'idrogeno. Anche altri scienziati ebbero dubbi etici. Ma la voglia di scoprire i misteri dell'universo, la complessità dell'atomo, erano un richiamo irresistibile nel loro mondo fatto di particelle che si scontrano. Fu forse la prima volta che il desiderio di conoscenza superò i dubbi etici e quindi quelli sul bene e sul male. La strada era aperta. Dopo la conoscenza dell'universo, lo sguardo è stato volto alla conoscenza dei segreti della vita. Nel 1953 Francis Crick ${ }^{4,5}$, James Watson $^{6}$ e Rosalind Franklin ${ }^{6}$ scoprirono la struttura molecolare dell'acido nucleico e il suo significato nella trasmissione delle informazioni negli esseri viventi, la famosa doppia elica del DNA. A rimarcare l'importanza di questa pietra miliare della vita fu inoltre la ferma critica di Crick del Young Earth Creationism ${ }^{7}$ che sosteneva che la terra e la vita sulla terra fossero opera della creazione divina avvenuta diecimila anni prima. Crick sostenne che la "Creation science" non poteva aver posto nelle aule della scuola pubblica. Craig Venter è un biologo americano noto per aver partecipato, con una società privata, la Celera Genomics, al sequenziamento del genoma dell'Homo sapiens con lo scopo di creare una banca dati genomica. Nel 2010 ha pubblicato su Science un articolo col quale comunicava di aver costruito in laboratorio la prima cellula artificiale controllata da un DNA sintetico ${ }^{8}$. La scienza nel percorso della conoscenza della vita ha imparato a leggere il DNA, a scriverlo e a modificarlo, tagliandone parti difettose con la tecnica CRISPR/Cas ${ }^{9}$ (Clustered Regularly Interspaced Short Palindromic Repeats) per sostituirle con sequenze corrette di DNA. Abbiamo capito che il DNA è, in concreto, un serie di informazioni che noi stiamo cominciando a capire e che il suo linguaggio è il campo prediletto dell'informatica in generale. Ma se con l'editing genetico possiamo evitare molte malattie, quali altre prospettive abbiamo davanti a noi? Siamo arrivati ad un punto in cui dobbiamo fermarci? Il prossimo passo, oltre alla creazione di embrioni ibridi di mammut e di organismi resistenti alle infezioni, alla modificazione di geni per frenare l'invecchiamento delle cellule e farle tornare giovani, come cerca di fare George Church $^{9}$, quale sarà? Forse quello di potenziare esponenzialmente le capacità umane, di creare superuomini? Siamo già andati oltre l'immaginario. Durante l'annuale International Summit on Human Genome Editing tenutosi ad Hong Kong, He Jiankui, un ricercatore cinese, ha annunciato di aver fatto nascere due gemelle da un padre sieropositivo, con il DNA modificato. Sostiene infatti di aver modificato il DNA di almeno una delle due gemelle neonate rendendola più resistente al virus dell'Hiv e, di conseguenza, all'eventuale contrazione dell'Aids. Per effettuare questa modifica del DNA sarebbe stato necessario disattivare il gene CCR5, con conseguenze al momento sconosciute. Ma il punto centrale sarà che questa modifica del DNA verrà trasmessa alle successive generazioni. Lo scienziato ha dato l'annuncio con un video su YouTube ripreso dalla rivista del Mit Technology Review, ed ha suscitato proteste in tutto il mondo, a partire dalla stessa Cina, dove oltre 120 scienziati hanno firmato una lettera in cui si definisce l'esperimento "una follia". Appunto. È necessario riprendere in mano il pensiero sulla conoscenza, sul bisogno di conoscere chi siamo, il senso da dare alla nostra vita, il nostro ruolo nell'universo. Ricominciare a considerare che la scienza deve progredire assieme al pensiero filosofico e a quello etico, nell'interesse della collettività. Ci manca, per sintetizzare, il pensiero critico che accompagni la scienza nell'anelito della conoscenza. Perché non possiamo trascurare di affrontare per tempo le sfide che stanno arrivando alla nostra comunità di uomini dall'intelligenza artificiale e dal pensiero sintetico.

\section{Dichiarazione di assenza di conflitto di interessi}

Gli Autori dichiarano di non avere conflitti di interessi.

\section{Finanziamenti}

Gli Autori dichiarano di non aver ricevuto finanziamenti specifici da qualsiasi ente nei settori pubblico, privato o senza fini di lucro.

\section{References}

1. Niccolò Copernico. De revolutionibus orbium coelestium. Norimbergae, apud Ioh. Petreium, 1543.

2. Sir Thomas L. Heath, Aristarchus of Samos - The Ancient Copernicus, A history of Greek astronomy to Aristarchus. Oxford, Clarendon, 1913.

3. Kai Bird e Martin J. Sherwin. Robert Oppenheimer, il padre della bomba atomica. $1^{\text {a }}$ ed. Milano, Garzanti Libri (Collezione storica), 2007.

4. Watson JD and Crick FH. Molecular Structure of Nucleic Acids; a structure for deoxyribose nucleic acid. Nature 1953; 171(4356):737-8.

5. Crick FH, Barnett L, Brenner $\mathrm{S}$ and Watts-Tobin RJ. General Nature Of The Genetic Code For Proteins. Nature, 1961; 192:1227-32. 
6. Watson JD and Crick FH. The Double Helix of DNA. Nature1953;

7. Numbers, Ronald. The Creationists: From Scientific Creationism to Intelligent Design. Harvard University Press 2006.
8. Gibson DG, Glass JI, Lartigue C, et al. Creation of a Bacterial Cell Controlled by a Chemically Synthesized Genome, Science 2010; 329(5987):52-6.

9. Nair P. (). Profile of George M. Church. Proceedings of the National Academy of Sciences. 2012; 109(30):11893-11895. 